\title{
Malignancy in a retrospective cohort of 17 patients with Dermatomyositis or Polymyositis in southern Tunisia
}

\author{
KHADIJA SELLAMI ${ }^{1}$, MADIHA MSEDDI ${ }^{1}$, MOUNA SNOUSSI ${ }^{2}$, HELA GHARBI ${ }^{1}$, \\ FATEN FRIKHA ${ }^{2}$, RAYDA BEN SALAH ${ }^{2}$, MOEZ JALLOULI' ${ }^{2}$, SAMEH MARZOUK ${ }^{2}$, \\ ABDERRAHMEN MASMOUDI ${ }^{1}$, ZOUHIR BAHLOUL ${ }^{2}$, HAMIDA TURKI $^{1}$ \\ ${ }^{1}$ Dermatology Department, Hedi Chaker University Hospital, Sfax, Tunisia \\ ${ }^{2}$ Internal Medicine Department, Hedi Chaker University Hospital, Sfax, Tunisia
}

\begin{abstract}
Introduction. The prognosis of dermatomyositis (DM)/ polymyositis (PM) in adults is partly related to their association with neoplasia. The aim of our study was to report the epidemiologic, clinical, paraclinical, therapeutic and evolutionary aspects of DM associated with malignancy in patients from Sfax, south eastern of Tunisia.

Methods. A retrospective cohort study of patients with DM or PM admitted in Dermatology and Internal Medicine Departments of Hedi Chaker University Hospital of Sfax between 1996 and 2015. Cases of DM or PM associated with malignancy were retained.

Results. Seventeen cases $(13.5 \%)$ of DM or PM associated with malignancy were noted. Fourteen patients had DM and 3 patients had PM. The Sex ratio M / F was 0.3 and the mean age at diagnosis was 56.5 years. In DM patients, malignancy preceded the myositis in $64.2 \%$ of cases. In PM patients, only one patient was known to have breast cancer and the myositis revealed the cancer for 2 others. Treatment consisted of corticosteroids associated with methotrexate in 4 cases. Outcome was fatal in 5 cases $(29.4 \%)$, due to the underlying cancer in 3 cases. Swallowing disorders related to $\mathrm{DM} / \mathrm{PM}$ were responsible for death in 2 cases.

Conclusion. There are no specific clinical or biological features in paraneoplastic DM. In our series, breast neoplasm represented the first cancer associated with DM. Cancers of nasopharynx, colon and urinary tract had the second position.
\end{abstract}

Keywords: dermatomyositis, polymyositis, cancer, paraneoplastic syndrome, risk.

\section{INTRODUCTION}

Dermatomyositis (DM) and polymyositis (PM) are autoimmune disorders characterized by inflammatory myopathy belonging to the group of connective tissue disorders. They are often idiopathic but may sometimes be associated with neoplasia. The association between DM and cancer has been reported since 1916 by Stertz [1]. Several studies since then had reported a 3 - to 6-fold increase in the risk of malignancy in patients with DM [2]. The association between cancer and myositis is widely variable in literature series. The present study aimed to evaluate the prevalence of malignancy in patients with DM/PM in Sfax (South-Eastern of Tunisia). We also studied the characteristics of the major type of the associated cancer, patient's age and gender and its prognosis. We emphasize on risk factors that must alert clinicians to search for underlying cancer, referring to our literature findings.
MATERIAL AND METHODS

We conducted a descriptive and retrospective study of all cases of paraneoplastic DM/PM followed at the Dermatology and Internal Medicine Departments of Hedi Chaker University Hospital (a tertiary care center) between 1996 and 2015. Diagnosis was based on Bohan and Peter criteria. We collected epidemiological, clinical, paraclinical, therapeutic and outcome data from registered medical observations.

\section{RESULTS}

During 20 years, 126 cases of DM/PM were diagnosed. Seventeen were paraneoplastic $(13.5 \%)$ distributed into 14 cases of DM and 3 cases of PM. For the whole group, at presentation, the mean age of patients was 56.5 years (range 34-83yr) and the sex ratio $\mathrm{M} / \mathrm{F}$ was 0.3 . Symptoms evolved for a mean period of 3.6 months (range: 1-23 months). 
In DM patients, mean age was 57.2 years (range: $34-83$ years) and the sex-ratio $\mathrm{M} / \mathrm{F}$ was 0.28 . The main clinical manifestations were: impaired general condition ( 4 cases, $28.5 \%$ ), erythrœedema of the face particularly the orbital rim (11 cases, 78\%), periungual erythema ( 5 cases, $36 \%$ ), Gottron's papules ( 1 case, $7 \%$ ), and myalgia with proximal muscle weakness (12 cases: $85.7 \%$ ). Skin necrosis was noted in only one patient and pruritus was present in 2 cases. Biological tests noted acceleration of erythrocyte sedimentation rate (ESR) (13 cases, 93\%) and elevated muscle enzymes (11 cases, 78\%). Elevated creatine phosphokinase (CPK) was constant except in 3 cases. Electromyography (EMG) findings were consistent with myositis in $64.2 \%$ of cases. Muscle biopsy performed in 5 patients confirmed the diagnosis in 4 cases. Two patients had amyopathic DM $(11.7 \%)$. The majority of patients (9 cases; $64.2 \%$ ) had been already diagnosed with neoplasia and the median time elapsed between discovery of cancer and diagnosis of DM was 2.8 years (range, 6 months - 12 years). Etiological investigations disclosed a latent malignancy in $35.7 \%$ of cases, mostly following the first year ( 8 cases, $57.2 \%$ ) or at the same time of diagnosis of DM (3 cases, $21.5 \%)$. Associated cancers were: breast cancer ( 6 cases, $42.8 \%$ ), nasopharynx carcinoma ( 2 cases, $14.2 \%$ ), colon carcinoma ( 2 cases, $14.2 \%$ ), urinary tract carcinoma( 2 cases, $14.2 \%$ ), ovary carcinoma (1 case, 7\%) and malignant thymomas (1 case, 7\%). After transient recovery of 10.6 months (range 224 months), DM recurred in 3 patients, announcing metastases (Table1).

In PM patients, only one patient had breast cancer diagnosed two years before PM. The diagnosis of malignancy followed the one of PM in two cases after a median time of 2 months (range, 1-3 months). Impairment of general condition and myalgia, with accelerated ESR and myolysis were constant. The diagnosis of PM was confirmed by EMG and muscle biopsy in the 3 patients. Associated cancers were breast carcinoma, gallbladder carcinoma and lymphoma (Table 2).

All patients were treated with corticosteroids at high doses and with methotrexate in 4 cases. A relapse was observed after initial improvement, in 3 cases of DM, and disclosed metastasis. Outcome was fatal in 5 cases $(29.4 \%)$, due to the underlying cancer in 3 cases. Swallowing disorders related to $\mathrm{DM} / \mathrm{PM}$ were responsible for death in 2 cases (Tables 1, 2).

\section{DISCUSSION}

DM and PM are rare inflammatory myopathies [3]. Their prognosis in adults is mainly determined by their association with neoplasia $[4,5]$. The association of DM with cancer is well established since 1916 [1, 3], whereas the relationship between $\mathrm{PM}$ and cancer is weaker [6] but it is becoming more common [5, 7]. Paraneoplastic DM's frequency is variously estimated through different series $[4,8]$. The incidence varies from 15 to $54 \%$ [4, 7-10]. In Tunisia, according to a multicenter retrospective study, it is estimated at $15 \%$ [10]. The incidence of cancer in DM is 5 to 7 times greater than that of the general population [7]. As in our study, the reported mean age of association myositis and cancer is 59.5 years [3] with a female predominance [10]. The risk of this association is higher among men over 50 years $[7,8,11]$. In children, this association has not been described yet. Paraneoplastic DM/PM has no specific clinical or paraclinical features [5]. In DM, cutaneous signs are always present and muscle symptoms appear simultaneously or later after skin involvement [5]. The neck and the back of the hands are electively involved [5]. Erythema is classic, appearing on outset or during the evolution of atrophy beaches. Zarrabi et al. reported a rare case of edematous paraneoplastic DM with florid subcutaneous edema [12]. In prolonged cases, poïkilo-DM can appear. DM associated with cancer is amyopathic in $22 \%$ of cases $[4,13,14]$, this association was noted in $11.7 \%$ of cases in our study. Some cases reported mucinosis as the only manifestation of DM $[15,16]$. Skin necrosis, observed only in one patient of our series, was reported in $62 \%$ of DM associated with cancer and in $7 \%$ of non-paraneoplastic DM [9]. High levels of muscle enzymes are common [9]. Elevated ESR, noted in $93 \%$ of our cases, was also noted in DM associated with cancer [5].

Poor prognostic factors in DM that must alert for the possibility of associated neoplasia are: older age (more than 50 years at diagnosis $[3,8,17,18]$ ), rapid onset, extensive skin lesions [19], pruritus [4], necrosis [6] and increased ESR [20]. Several studies have also found that the presence of certain antibodies in myositis such as anti JO1, anti Mi2 and anti U1RNP is associated with a low risk of cancers [21]. Nevertheless, the anti-155/140 antibodies are associated with a higher risk of cancer in DM [22]. It was recently established that anti-SAE1, and previously reported anti-TIF1- $\gamma$ and anti-NXP2 
antibodies, are all associated with an increased risk of cancer in patients with DM/PM. It could be a tool for etiologic research of paraneoplastic DM/
PM [23]. Unfortunately, these laboratory tests were not performed in our study because of the lack of suitable kit.

Table 1

Baseline patients characteristics in DM patients

\begin{tabular}{|c|c|c|c|c|c|c|c|c|c|}
\hline Patients & Sex & $\begin{array}{c}\text { Age } \\
\text { (years) }\end{array}$ & $\begin{array}{l}\text { Interval between } \\
\text { the diagnosis of } \\
\text { PM and the } \\
\text { associated cancer } \\
\end{array}$ & Cutaneous involvement & $\begin{array}{l}\text { Other systemic } \\
\text { involvements }\end{array}$ & $\begin{array}{l}\text { Biological } \\
\text { findings }\end{array}$ & $\begin{array}{c}\text { Type of } \\
\text { malignancy }\end{array}$ & Treatment & Outcome \\
\hline Patient 1 & $\mathrm{~F}$ & 49 & 96 mo after & $\begin{array}{c}\text { Periorbital erythroedema } \\
\text { Erythema of the trunk and } \\
\text { upper limbs } \\
\text { Periungual erythema }\end{array}$ & $\begin{array}{l}\text { Myalgia with } \\
\text { proximal muscle } \\
\text { weakness }\end{array}$ & $\begin{array}{c}\text { ESR 40 } \\
\text { CPK 279 } \\
\text { LDH 280 } \\
\text { ANA }(+)\end{array}$ & $\begin{array}{c}\text { Breast } \\
\text { carcinoma }\end{array}$ & $\begin{array}{c}\text { High-dose } \\
\text { corticosteroids }\end{array}$ & Favorable \\
\hline Patient 2 & $\mathrm{~F}$ & 68 & 24 mo before & $\begin{array}{l}\text { Periorbital erythroedema } \\
\text { Erythema of sun-exposed } \\
\text { areas; Face telangiectasia }\end{array}$ & $\begin{array}{l}\text { Myalgia with } \\
\text { proximal muscle } \\
\text { weakness }\end{array}$ & $\begin{array}{c}\text { ESR } 80 \\
\text { CPK 500 } \\
\text { LDH } 809 \\
\text { ANA }(+) \\
\end{array}$ & $\begin{array}{c}\text { Nasopharynx } \\
\text { carcinoma }\end{array}$ & $\begin{array}{c}\text { High-dose } \\
\text { corticosteroids }+ \\
\text { Methotrexate }\end{array}$ & Favorable \\
\hline Patient 3 & M & 51 & Concomitant & $\begin{array}{l}\text { Periorbital oedema } \\
\text { Erythema of legs and } \\
\text { proximal interphalangeal } \\
\text { joints }\end{array}$ & $\begin{array}{c}\text { Effort-related } \\
\text { fatigability } \\
\text { Arthralgia, } \\
\text { myalgia, proximal } \\
\text { muscle weakness }\end{array}$ & $\begin{array}{l}\text { ESR } 53 \\
\text { CPK } 114 \\
\text { LDH } 350 \\
\text { ANA }(+)\end{array}$ & $\begin{array}{c}\text { Urinary tract } \\
\text { carcinoma }\end{array}$ & $\begin{array}{c}\text { High-dose } \\
\text { corticosteroids }+ \\
\text { Methotrexate }\end{array}$ & \begin{tabular}{|c|} 
Relapse after \\
transient \\
improvement \\
(metastasis)
\end{tabular} \\
\hline Patient 4 & M & 68 & 12 mo before & $\begin{array}{c}\text { Erythema of the face and the } \\
\text { low-neckline } \\
\text { Periungual erythema }\end{array}$ & $\begin{array}{l}\text { Effort-related } \\
\text { fatigability } \\
\text { Dyspnea, cough } \\
\text { Swallowing } \\
\text { disorders }\end{array}$ & $\begin{array}{c}\text { ESR 63 } \\
\text { CPK 5241 } \\
\text { LDH 591 } \\
\text { ANA }(+)\end{array}$ & $\begin{array}{c}\text { Colon } \\
\text { carcinoma }\end{array}$ & $\begin{array}{c}\text { High-dose } \\
\text { corticosteroids }+ \\
\text { Methotrexate }\end{array}$ & 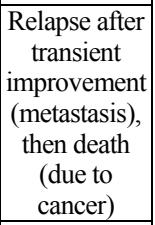 \\
\hline Patient 5 & $\mathrm{~F}$ & 60 & 12 moafter & \begin{tabular}{|} 
Erythema of the low- \\
neckline and the upper limbs
\end{tabular} & $\begin{array}{l}\text { Effort-related } \\
\text { fatigability } \\
\text { Proximal muscle } \\
\text { weakness }\end{array}$ & \begin{tabular}{|c|} 
ESR 11 \\
CPK 34 \\
LDH 202 \\
ANA (+)
\end{tabular} & $\begin{array}{l}\text { Breast } \\
\text { carcinoma }\end{array}$ & $\begin{array}{c}\text { High-dose } \\
\text { corticosteroids }\end{array}$ & Favorable \\
\hline Patient 6 & M & 59 & 24 mo after & $\begin{array}{l}\text { Erythema of the face, the } \\
\text { upper limbs and the trunk }\end{array}$ & $\begin{array}{l}\text { Proximal muscle } \\
\text { weakness }\end{array}$ & $\begin{array}{l}\text { ESR 14 } \\
\text { CPK 64 } \\
\text { LDH NR } \\
\text { ANA NR }\end{array}$ & $\begin{array}{c}\text { Nasopharynx } \\
\text { carcinoma }\end{array}$ & $\begin{array}{c}\text { High-dose } \\
\text { corticosteroids }\end{array}$ & Favorable \\
\hline Patient 7 & $\mathrm{~F}$ & 51 & 120 mo after & $\begin{array}{l}\text { Periorbital erythema } \\
\text { Erythema of upper and } \\
\text { lower limbs }\end{array}$ & $\begin{array}{l}\text { Impaired general } \\
\text { condition } \\
\text { Polyarthralgia }\end{array}$ & $\begin{array}{c}\text { ESR } 17 \\
\text { CPK } 1105 \\
\text { LDH } 2134 \\
\text { ANA (-) } \\
\end{array}$ & $\begin{array}{l}\text { Breast } \\
\text { carcinoma }\end{array}$ & $\begin{array}{c}\text { High-dose } \\
\text { corticosteroids }\end{array}$ & Favorable \\
\hline Patient 8 & $\mathrm{~F}$ & 83 & Concomitant & $\begin{array}{l}\text { Erythroedema of the face } \\
\text { and the low-neckline }\end{array}$ & Myalgia & $\begin{array}{c}\text { ESR 38 } \\
\text { CPK } 7504 \\
\text { LDH NF } \\
\text { ANA }(+)\end{array}$ & $\begin{array}{l}\text { Ovary } \\
\text { carcinoma }\end{array}$ & $\begin{array}{c}\text { High-dose } \\
\text { corticosteroids }\end{array}$ & $\begin{array}{c}\text { Death (due to } \\
\text { cancer) }\end{array}$ \\
\hline Patient 9 & M & 50 & Concomitant & $\begin{array}{l}\text { Erythroedema of the face } \\
\text { and the upper limbs }\end{array}$ & $\begin{array}{c}\text { Proximal muscle } \\
\text { weakness } \\
\text { Swallowing } \\
\text { disorders } \\
\end{array}$ & $\begin{array}{c}\text { ESR } 60 \\
\text { CPK } 1848 \\
\text { LDH } 600 \\
\text { ANA (-) } \\
\end{array}$ & $\begin{array}{c}\text { Neuroendocri } \\
\text { ne carcinoma } \\
\text { of the colon }\end{array}$ & $\begin{array}{c}\text { High-dose } \\
\text { corticosteroids }\end{array}$ & Favorable \\
\hline Patient 10 & $\mathrm{~F}$ & 44 & 12 mo after & \begin{tabular}{|c|} 
Eczema-like eruption of the \\
face and the trunk \\
Periungual erythema \\
Gottron's papules \\
\end{tabular} & $\begin{array}{l}\text { Proximal muscle } \\
\text { weakness }\end{array}$ & $\begin{array}{c}\text { ESR } 86 \\
\text { CPK 502 } \\
\text { LDH 330 } \\
\text { ANA }(+) \\
\end{array}$ & $\begin{array}{c}\text { Breast } \\
\text { carcinoma }\end{array}$ & $\begin{array}{c}\text { High-dose } \\
\text { corticosteroids }\end{array}$ & Favorable \\
\hline Patient 11 & $\mathrm{~F}$ & 59 & 24 mo after & $\begin{array}{c}\text { Erythema of sun-exposed } \\
\text { areas }\end{array}$ & $\begin{array}{c}\text { Myalgia with } \\
\text { proximal muscle } \\
\text { weakness }\end{array}$ & $\begin{array}{c}\text { ESR 32 } \\
\text { CPK 225 } \\
\text { LDH } 548 \\
\text { ANA (-) } \\
\end{array}$ & $\begin{array}{l}\text { Breast } \\
\text { carcinoma }\end{array}$ & $\begin{array}{c}\text { High-dose } \\
\text { corticosteroids }\end{array}$ & Favorable \\
\hline Patient 12 & $\mathrm{~F}$ & 34 & 6 mo after & $\begin{array}{l}\text { Erythema of the hands, } \\
\text { low-neckline and loins }\end{array}$ & $\begin{array}{l}\text { Proximal muscle } \\
\text { weakness }\end{array}$ & $\begin{array}{l}\text { ESR 33 } \\
\text { CPK NR } \\
\text { LDH NR } \\
\text { ANA NR } \\
\end{array}$ & Thymoma & $\begin{array}{c}\text { High-dose } \\
\text { corticosteroids }\end{array}$ & Favorable \\
\hline Patient 13 & $\mathrm{~F}$ & 53 & 12 mo after & $\begin{array}{c}\text { Periorbital erythema } \\
\text { Erythema of limbs extension } \\
\text { face }\end{array}$ & $\begin{array}{c}\text { Asthenia } \\
\text { Myalgia } \\
\text { Proximal muscle } \\
\text { weakness }\end{array}$ & $\begin{array}{l}\text { ESR } 19 \\
\text { CPK NR } \\
\text { LDH NR } \\
\text { ANA NR }\end{array}$ & $\begin{array}{l}\text { Breast } \\
\text { carcinoma }\end{array}$ & $\begin{array}{c}\text { High-dose } \\
\text { corticosteroids }\end{array}$ & $\begin{array}{c}\text { Death } \\
\text { (due to DM) }\end{array}$ \\
\hline Patient 14 & $\mathrm{~F}$ & 72 & 24 mo before & $\begin{array}{c}\text { Erythema of sun-exposed } \\
\text { areas and limbs extension } \\
\text { face }\end{array}$ & $\begin{array}{l}\text { Impaired general } \\
\text { condition }\end{array}$ & $\begin{array}{l}\text { ESR NR } \\
\text { CPK NR } \\
\text { LDH NR } \\
\text { ANA NR } \\
\end{array}$ & $\begin{array}{c}\text { Urinary tract } \\
\text { carcinoma }\end{array}$ & $\begin{array}{c}\text { High-dose } \\
\text { corticosteroids }\end{array}$ & \begin{tabular}{|c|} 
Relapse after \\
rtransient \\
improvement \\
(metastasis)
\end{tabular} \\
\hline
\end{tabular}

Abbreviations: F: female; M: male; ESR: Erythrocyte sedimentation rate; CPK: Creatine phosphokinase; LDH: Lactate dehydrogenase; ANA: Antinuclear antibodies; NR datum not recorded. Mo: months 
Table 2

Baseline patients characteristics in PM patients

\begin{tabular}{|l|c|c|c|c|c|c|c|c|}
\hline Patients & Sex & $\begin{array}{c}\text { Age } \\
\text { (years) }\end{array}$ & $\begin{array}{c}\text { Interval between the } \\
\text { diagnosis of PM and the } \\
\text { associated cancer }\end{array}$ & $\begin{array}{c}\text { Biological } \\
\text { findings }\end{array}$ & $\begin{array}{c}\text { Other systemic } \\
\text { involvement }\end{array}$ & Malignancy & Treatment & Outcome \\
\hline Patient 1 & F & 39 & 24 mo after & $\begin{array}{c}\text { ESR 110 } \\
\text { CPK 1303 } \\
\text { LDH 1850 } \\
\text { ANA NR }\end{array}$ & Asthenia & Breast carcinoma & $\begin{array}{c}\text { High-dose } \\
\text { corticosteroids }\end{array}$ & Death (due to cancer) \\
\hline Patient 2 & F & 61 & Concomitant & $\begin{array}{c}\text { ESR 115 } \\
\text { CPK 1407 } \\
\text { LDH 979 } \\
\text { ANA (-) }\end{array}$ & $\begin{array}{c}\text { Asthenia } \\
\text { Weight loss } \\
\text { Myocarditis }\end{array}$ & $\begin{array}{c}\text { Gallbladder } \\
\text { carcinoma }\end{array}$ & $\begin{array}{c}\text { High-dose } \\
\text { corticosteroids + } \\
\text { Methotrexate }\end{array}$ & Death (due to PM) \\
\hline Patient 3 & F & 60 & 12 mo before & $\begin{array}{c}\text { ESR 30 } \\
\text { CPK 45 } \\
\text { LDH 1160 } \\
\text { ANA (+) }\end{array}$ & Arthralgia & Lymphoma & $\begin{array}{c}\text { High-dose } \\
\text { corticosteroids }\end{array}$ & Relapse after transient \\
improvement (metastasis)
\end{tabular}

Abbreviations: F: Female; M: Male; ESR: Erythrocyte sedimentation rate; CPK: Creatine phosphokinase; LDH: Lactate dehydrogenase; ANA: Antinuclear antibodies; NR: datum not recorded. Mo: months

Table 3

$\mathrm{DM} / \mathrm{PM}$ and type of associated malignancy in different populations

\begin{tabular}{|c|c|c|c|c|c|c|}
\hline Study & Population & Study period & $\begin{array}{c}\text { Number of } \\
\text { patients }\end{array}$ & Sex/Age & (n) Malignancy & Types of malignancies \\
\hline $\begin{array}{c}\text { Stockton et al. } \\
{[18]}\end{array}$ & Scotland & $1982-1996$ & 705 & $\mathrm{~F}(433) / \mathrm{M}(272)$ & 50 & $\begin{array}{l}\text { Oesophagus (1); stomach (3); colon (7); lung (19); } \\
\text { breast (3); cervix uteri (2); ovary (2); prostate (1); } \\
\text { bladder (1); unspecified (6); Hodgkin's disease (1); } \\
\text { Non-Hodgkin's lymphoma (2) }\end{array}$ \\
\hline $\begin{array}{c}\text { Chen et al. } \\
\text { [25] }\end{array}$ & Taiwan & $1997-2007$ & 1655 & $\mathrm{~F}(1141) / \mathrm{M}(514)$ & 95 & $\begin{array}{l}\text { Nasopharynx (30); lung (22); } \\
\text { breast (9); uterine cervix } \\
\text { (3); ovary (2); lymphoma/ } \\
\text { leukemia (4); oropharynx } \\
\text { and larynx (1); esophagus (1); } \\
\text { liver/gallbladder; colorectum } \\
\text { (5); stomach (1); pancreas } \\
(1) ; \text { kidney (3); urinary } \\
\text { bladder (2); melanoma (1); } \\
\text { bone/joint (1); thyroid (2); } \\
\text { metastatic cancers (2) }\end{array}$ \\
\hline $\begin{array}{c}\text { Limaye et al. } \\
{[27]}\end{array}$ & $\begin{array}{c}\text { South } \\
\text { Australia }\end{array}$ & 1980-2009 & 373 & $\mathrm{~F}(245) / \mathrm{M}(179)$ & 43 & $\begin{array}{l}\text { lung cancers (7), } \\
\text { prostate cancers (6), colon cancers (4), lymphomas } \\
\text { (4), Hodgkin's disease (2), leukaemias (3), breast (3), } \\
\text { melanomas( } 2), \text { skin cancers (3), bladder ( 3) } \\
\text { endometrial ( } 1) \text { testis }(1), \text { unspecified, site (1), } \\
\text { 1nasopharyngeal( } 1) \text {, pancreatic (1) and } \\
\text { myelodysplastic syndrome (1) }\end{array}$ \\
\hline $\begin{array}{c}\text { Requena et al. } \\
{[37]}\end{array}$ & Spain & $1994-2013$ & 12 & $\mathrm{~F}(6) / \mathrm{M}(6)$ & 12 & $\begin{array}{l}\text { Ovarian Cancer (3), Bladder cancer (3), Lung cancer } \\
\text { (1), stomach (1), breast cancer (1), Prostate (1) and } \\
\text { cervix (1), Non-Hodgkin lymphoma (1) }\end{array}$ \\
\hline Our study & South Tunisia & $1996-2015$ & 126 & $\mathrm{~F}(93) / \mathrm{H}(33)$ & 17 & $\begin{array}{l}\text { Breast carcinoma (7), Gallbladder carcinoma (1), } \\
\text { Lymphoma (1), Thymoma (1), Urinary tract } \\
\text { carcinoma (2), Colon carcinoma (2), Nasopharynx } \\
\text { carcinoma (2), Ovary carcinoma (1) }\end{array}$ \\
\hline
\end{tabular}

$\mathrm{DM} / \mathrm{PM}$ could follow an already diagnosed cancer, as 10 of our patients, but it often precedes cancer by several months $[17,18]$. The risk of cancer occurrence seems to be greater in the first year of DM/PM diagnosis [3, 6, 11, 24]. Associated cancers do not seem to differ from those frequent in general population [10]. In general, carcinomas are more frequent than sarcomas and lymphoproliferative tumors. These cancers are dominated by breast cancer in women (41\% in our series) $[7,9,25]$ and lung cancers in men $[7,9]$. According to Hendren et al., the presence of DM is associated with moreadvanced breast cancer stage and is most commonly associated with invasive ductal carcinoma [26]. Other cancers were reported especially colorectal cancers, cancers of the ovary [3], the nasophagynx, the prostate $[27,28]$. But, all localizations are possible: kidney, thyroid, malignant thymoma or lymphoma. Cancers of unknown primary-related DM had also been reported [29]. Zhang et al. reported a rare case of occult breast cancer in a man associated to DM [30]. To date and to our knowledge related to 
skin cancers, yet so common, had not been reported (Table 3: DM/PM and type of associated malignancy in different populations).

There is often a clear correlation between changes in DM and those of the associated cancer [27]. For some patients, cancer treatment results in DM improvement or even recovery from DM [33]. If metastases appear, DM may recur. The latter situation was noted in 3 of our patients. DM was reported in association to a recurrence of breast cancer [25].

Disclosure of a cancer in a patient with DM is a poor prognostic factor. Mortality ranges from 35 to $58 \%$ after 20 months and 7 years, respectively [10]. It reaches $100 \%$ in case of severe forms of DM [4]. DM may be fatal, by swallowing disorders associated with muscle impairment as in 2 of our patients, but death is linked to cancer in $90 \%$ of cases [10].

Etiopathogenesis of this paraneoplastic syndrome remains controversial $[6,7]$. Some authors propose an alteration of the extracellular matrix of the skin by increased inflammatory and immunological reaction in patient's skin. DM and some cancers share etiologic factors [6].

As the pathophysiology of paraneoplastic $\mathrm{DM} / \mathrm{PM}$ remains unclear, no standard treatment regimen has been established yet [34]. High-dose corticosteroids are suggested as the first-line therapy. It was the therapeutic choice for all our patients. Immunosuppressant drugs or IV human immunoglobulins are suggested as second-line therapy. Drugs administered in case of DM do not seem to increase the risk of cancers [6]. Searching for neoplasia in DM is discussed [3], this assessment is systematic in adults for some authors [9], mainly if they had a history of neoplasia, rapid onset of DM, recurrence or sudden worsening of previously stable DM [24]. Some authors recommended repeated investigations at regular intervals of 6 to 12 months after diagnosis to prevent discovery of cancer at advanced stage [35]. Gkegkes et al. had even suggested to perform systematic colonoscopy in these patients [36].

\section{CONCLUSION}

In adult patients with $\mathrm{DM} / \mathrm{PM}$, the presence of predictive factors such as advanced age, impairment of general condition and accelerated ESR must alert clinicians to the possibility of associated neoplasia. Thorough physical examination and accurate investigations are needed to detect cancer at an early stage. In our series, breast neoplasm represents the first cancer associated with DM. Cancers of nasopharynx, colon and urinary tract have the second position. Our study confirms correlation between neoplasia and DM's outcomes. The limitations of our study are the small number of patients with cancer and it would have been interesting to make comparisons with $\mathrm{DM} / \mathrm{PM}$ patients that had no neoplasias and search for some distinct characteristics.

Conflict of interest.The authors declare that there are not conflicts of interest.

Introducere. Prognosticul dermatomiozitei (DM)/polimiozitei (PM) la adult este legată şi de asocierea cu neoplaziile. Scopul studiului a fost de a raporta caracteristicile clinice, epidemiologice, paraclinice, terapeutice şi de evoluție ale pacienților din Sfax, sud-estul Tunisiei, care aveau DM şi asociau şi malignități.

Materiale. A fost realizat un studiu retrospectiv (1996-2015) pe pacienții cu DM sau PM internați în clinicile de Dermatologie şi Medicină Internă ale Spitalului Universitar Hedi Chaker din Sfax. Au fost analizate cazurile care aveau diagnosticul de DM sau PM şi neoplazie.

Rezultate. 17 cazuri (13.5\%) din pacienții cu DM sau PM aveau asociere cu malignitate. 14 pacienți au avut DM şi 3 pacienți au avut PM. Raportul de sex masculin/feminin a fost de 0.3 şi media de vârstă a diagnosticului a fost la 56.5 ani. La pacienții cu DM malignitatea a precedat miozita în $64.2 \%$ din cazuri. La pacienții cu PM numai un pacient avea la momentul diagnosticului cancer de sân. Tratamentul a constat în corticoterapie asociat cu methotrexat la 4 pacienți. La 5 pacienți s-a înregistrat deces- 3 dintre acestea datorate cancerului şi 2 datorate tulburărilor de deglutiție. 
Concluzii. Nu s-au evidențiat caracteristici specifice clinice sau biologice la pacienții cu DM paraneoplazică. La pacienții analizați, cancerul de sân a fost primul asociat cu DM. Cancerul nazofaringelui, colonului şi ale tractului urinar au urmat ca frecvență.

Correspondence to: Dr. Khadija Sellami, MD, Hedi Chaker University Hospital, Elain Road 2 Km, 3003 Sfax, Tunisia Phone: 0021655218979

E-mail: Khadija@sellami.com

\section{REFERENCES}

1. STERTZ G. Polymyositis. BerlKlinWochenschr 1916; 53:489.

2. FARDET L, DUPUY A, GAIN M, KETTANEH A, CHERIN P, BACHELEZ H, et al. Factors associated with underlying malignancy in a retrospective cohort of 121 patients with dermatomyositis. Medicine (Baltimore). 2009 Mar; 88(2):91-7.

3. GAINON J, BART PA, WAEBER G. Can we predict the risk of malignancy with Dermatomyositis. Schweiz Rundsch Med Prax 2003; 92: 1734-9.

4. GALLAIS V, CRICKX B, BELAICH S. Facteurs pronostiques et signes prédictifs de cancer au cours de la dermatomyosite de l'adulte. Ann DermatolVenereol 1996; 123: 722-6.

5. AMERIO P, GIRARDELLI CR, PROIETTO G, FORLEO P, CERRITELLI L, FELICIANI C, COLONNA L, et al. Usefulness of erythrocyte sedimentation rate as tumor marker in cancer associated dermatomyositis. Eur J Dermatol 2002; 12:165-9.

6. AIRIO A, PUKKALA E, ISOMAKI H. Evaluated cancer incidence in patients with dermatomyositis: a population based study. J Rhumatol 1995; 22:1300-3.

7. DOURMISHEV LA. Dermatomyositis associated with malignancy 12 case reports. Adv Exp Med Biol 1999; 455:193-9.

8. LEOW YH, GOH CL. Malignancy in adult dermatomyositis. Int J Dermatol 1997; 36:904-7.

9. BURNOUF M, MAHE E, VERPILLAT P, DESCAMPS V, LEBRUN-VIGNES B, PICARD-DAHAN C, et al. Les nécroses cutanées dans les dermatomyosites de l'adulte sont prédictives de l'association à une néoplasie. Ann Dermatol Venereol 2003; 130: 313-6.

10. MEBAZAA A, BOUSSEN H, NOUIRA R, ROKBANI L, BEN OSMAN-DHAHRI A, BOUAOUINA N, et al. Dermatomyositis and malignancy: a multicenter national retrospective study of 20 cases. J Am Acad Dermatol 2003: 48:530-4.

11. WAKATA N, KURIHARA T, KINISHITA M. Polymyositis and dermatomyositis associated with malignancy. Int J Dermatol 2002; 41:729-34.

12. ZARRABI K, CHOY T, SWEENEY K, DESAI V, KERESZTES R. Paraneoplastic edematous dermatomyositis: A rare syndrome observed in a case of small cell lung cancer. Clin Pract. 2017 Oct 23; 7(4):982.

13. FINGER DR, DUNN CL, GILLILAND WR, JAMES WD. Amyopathic dermatomyositis associated with malignancy. Int J Dermatol 1996; 35:663-4.

14. LANGAN SM, O'BRIAN S, BARNES L. Dermatomyositis associated with angiotropic lymphoma. Clin Exp Dermatol 2003; 28:597-9.

15. TAN E, TAN SH, NGSK. Cutaneous mucinisis in dermatomyositis associated with a malignant tumor. J Am Acad Dermatol 2003; 48:S41-2.

16. MAHE E, DESCAMPS V, BURNOUF M, CRICKX B. A helpful clinical sign predictive of cancer in adult dermatomyositis: cutaneous necrosis. Arch Dermatol 2003; 139:539.

17. ANZAI S, KATAGIRI H, SATO T, TAKAYASU S. Dermatomyositis associated with primary intramuscular B cell lymphoma. J Dermatol 1997; 24:649-53.

18. LUU X, LEONARD S, JOSEPH KA. Dermatomyositis presenting as a paraneoplastic syndrome with resolution of symptoms following surgical management of underlying breast malignancy. J Surg Case Rep. 2015 Jul 7; 2015(7). pii: rjv075.

19. STOCKTON D, DOHERTY VR, BREWSTER DH. Risk of cancer in patients with dermatomyositis or polymyositis, and follow-up implication: a Scottish population-based cohort study. Br J Cancer 2001; 85:41-5.

20. CALLEN P. When and how should the patient with dermatomyositis or amyopathic dermatomyositis be assessed for possible cancer? Arch Dermatol 2002; 138:969-71.

21. MADAN V, CHINOY H, GRIFFITHS CE, COOPER RG. Defining cancer risk in dermatomyositis. Part II. Clin Exp Dermatol. 2009; 34:561-5.

22. BIELSA I. Nuevos autoanticuerpos en la dermatomiositis. Actas Dermosifiliogr. 2009; 100:182-9.

23. YANG H, PENG Q, YIN L, LI S, SHI J, ZHANG Y, et al. Identification of multiple cancer-associated myositis-specific autoantibodies in idiopathic inflammatory myopathies: a large longitudinal cohort study. Arthritis Res Ther. 2017 Nov 25; 19(1):259.

24. CELEBI S, GUL A, KAMALI S, INANC L, ARAL O, KONICE M. Amyopathic dermatomyositis associated with cervical cancer. Clin Rheumatol 2001; 20:438-40.

25. GOYAL S, NOUSARI HC. Paraneoplastic amyopathic dermatomyositis associated with breast cancer recurrence. J Am Acad Dermatol 1999; 41:874-5. 
26. HENDREN E1, VINIK O2, FARAGALLA H3, HAQ R4. Breast cancer and dermatomyositis: a case study and literature review. Curr Oncol. 2017 Oct; 24(5):e429-e433.

27. MASUDA H, URUSHIBARA M, KIHARA K. Successful treatement of dermatomyositis associated with adenocarcinoma of the prostate after prostatectomy. J Urol 2003, 169:1084.

28. ABRAHAM Z, ROSNER I, ROZENBAUM M, GOLDENBERG K, WELLER B. Dermatomyositis and nasopharyngeal carcinoma. J Dermatol 1998; 25:539-43.

29. SONNENBLICK A. Carcinoma of unknown primary and paraneoplastic dermatomyositis. World J Clin Oncol. 2015 Dec 10; 6(6):295-8.

30. ZHANG L, ZHANG C, YANG Z, HE M, ZHANG L, EZZAT S, et al. Male occult triple-negative breast cancer with dermatomyositis: a case report and review of the literature. Onco Targets Ther. 2017 Nov 14; 10:5459-5462.

31. CHEN YJ, WU CY, HUANG YL, WANG CB, SHEN JL, CHANG YT. Cancer risks of dermatomyositis and polymyositis: a nationwide cohort study in Taiwan. Arthritis Res Ther. 2010; 12(2):R70.

32. LIMAYE V, LUKE C, TUCKER G, et al. The incidence and associations of malignancy in a large cohort of patients with biopsy determined idiopathic inflammatory myositis. Rheumatol Int. 2013; 33(4):965-971.

33. KUSUNOKI T, HOMMA H, KIDOKORO Y, YANAI A, IKEDA K, WADA R. A Case of Laryngeal Cancer Associated with Dermatomyositis. Clin Pract. 2015 Dec 22; 5(4):818.

34. PAPAKONSTANTINOU E1, KAPP A2, RAAP U3. A mild form of dermatomyositis as a prodromal sign of lung adenocarcinoma: a case report. J Med Case Rep. 2016 Feb 6; 10(1):34.

35. LAMQUAMI S, ERRARHAY S, MAMOUNI N, BOUCHIKHI C, BANANI A. Dermatomyositis revealing breast cancer: report of a case. Pan Afr Med J. 2015 Jun 5; 21:89.

36. GKEGKES ID, MINIS EE, IAVAZZO C. Dermatomyositis and colorectal cancer: a systematic review. Ir J Med Sci. 2017 Nov 22.

37. REQUENA C1, ALFARO A2, TRAVES V et al. Paraneoplastic dermatomyositis: a study of 12 cases. Actas Dermosifiliogr. 2014 Sep; 105(7):675-82.

Received February 25, 2018 